\title{
A strategy for regional-scale FWI in the salt provinces offshore Brazil
}

\author{
Juergen Fruehn*, Stuart Greenwood, Ross O'Driscoll, lan Jones and John Brittan, ION
}

Copyright 2019, SBGf - Sociedade Brasileira de Geofísica

This paper was prepared for presentation during the $16^{\text {th }}$ International Congress of the Brazilian Geophysical Society held in Rio de Janeiro, Brazil, 19-22 August 2019.

Contents of this paper were reviewed by the Technical Committee of the $16^{\text {th }}$ International Congress of the Brazilian Geophysical Society and do not necessarily represent any position of the SBGf, its officers or members. Electronic reproduction or storage of any part of this paper for commercial purposes without the written consent of the Brazilian Geophysical Society is prohibited.

\section{Introduction}

Salt imaging is a great challenge for model builders, as it is often associated with near vertical dips and the cumbersome interpretation of overhangs. It is common practice to use reverse time migration (RTM) to optimize the picking of the salt geometry, and more recently, to use full-waveform inversion (FWI) to facilitate the construction of reliable and robust parameter models.

However, as is now relatively well known, no one technique alone can deliver the required robust parameter models. In this paper, we present a strategy for the stepwise use of FWI, employing both the transmitted (refraction) and reflected wavefields, in conjunction with different minimization norms, to facilitate the avoidance of cycle-skipping issues, in an attempt to mitigate the risk of convergence to local (rather than hopefully global) inversion minima.

\section{Background}

The study area considered here is to the east of the presalt polygon in the Campos basin offshore Brazil (Figure 1 ), where domes of highly mobilized salt are widespread (Pavlov et al., 2018). Great interest in this area has recently re-energized the E\&P community, after the Brazilian government opened the area for international exploration. We show examples from a reprocessing program utilizing publically available $3-D$ seismic data which had the objectives of developing a basin-wide, unified PreSDM image, covering an area in excess of $100,000 \mathrm{sqkm}$. The FWI workflow discussed here is from a 12,500 sqkm subset, for which Figure 2 shows a segment from typical seismic section.

The main imaging objective of the program is to build the best possible overall image, which will then allow a largescale re-evaluation of the hydrocarbon potential at all possible levels, from the overhanging salt domes of the eastern areas to the deeper targets trapped by carbonates in the central and western areas and the very deep sub-salt regions. This is a very challenging task, as the thickness of salt bodies varies greatly from a few dozen metres to 3-4 kilometres, with added local complications such as overhangs, 'dirty salt', and fast carbonates capping or abutting the salt. The general strategy for model-building of such complicated salt bodies is to undertake a staged approach (Jones and Davison 2014).

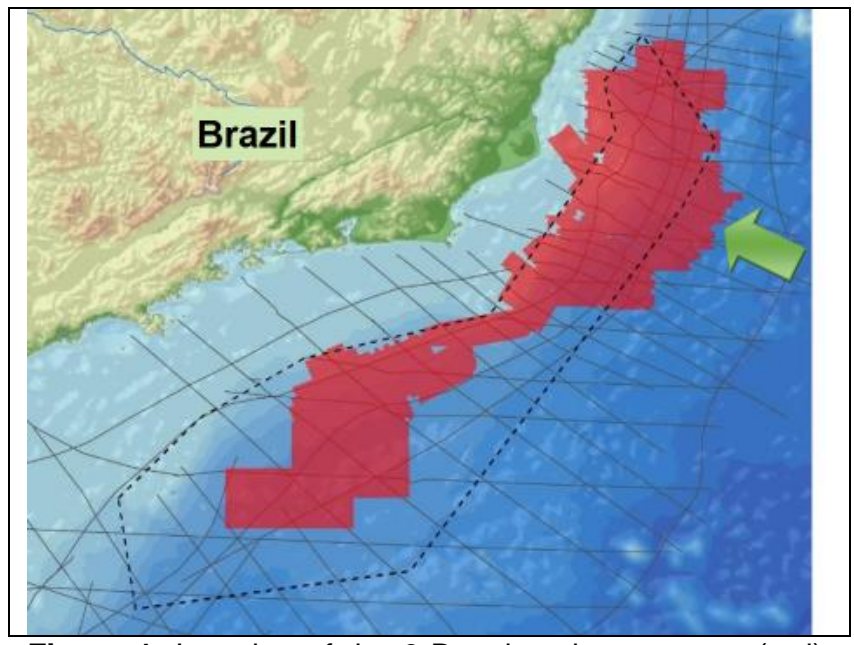

Figure 1. Location of the 3-D re-imaging program (red), Pre-Salt polygon (black dashed line), extension area (green arrow)

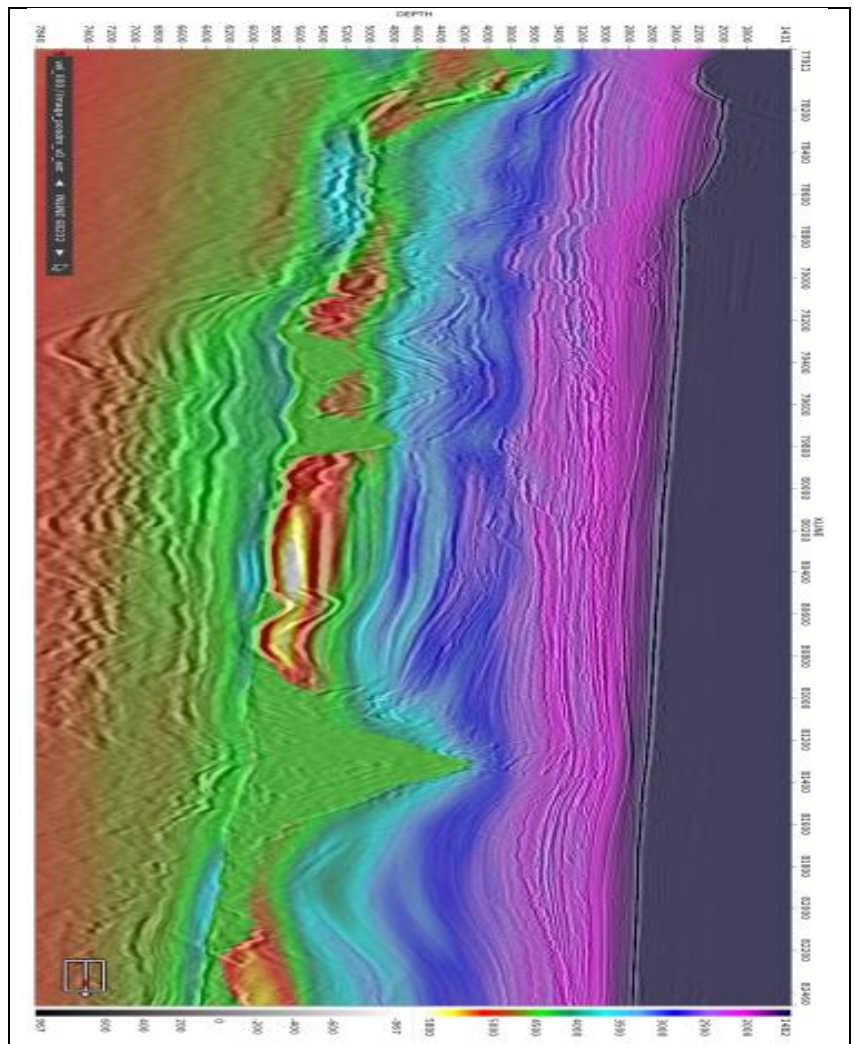

Figure 2. Typical seismic depth image with tomographic model velocity overlay: the shallow overburden in the deep water area is relatively benign, but the complex salt structures are interspersed between high velocity carbonate bodies both of which are immediately underlain by a lower velocity regime with hydrocarbon-bearing potential. 


\section{Model Building Strategy}

For an FWI approach, care must be taken with the choice of the starting models so as not to get 'stuck' in local minima during the inversion process. In the context of seismic data, this issue relates to the cycle-skipping problem (e.g. Warner et al., 2013; Jones 2018). In the simplest forms of $\mathrm{FWI}$, the minimization norm being used will be that of least-squares, which has very good model resolution, but suffers most from the likelihood of converging to a local, rather than a global minimum.

In order to facilitate avoidance of cycle-skipping pitfalls, several authors have proposed alternative norms (e.g. Warner and Guasch 2016; Jiao et al., 2015; Vigh et al., 2017; Schuster 2017; Yang and Engquist 2018; Wang et al., 2018). In addition to the choice of the norm, we also need to distinguish between the use of the transmitted (refracted) and the reflected wavefields. The transmitted wavefield is of most use in updating the very shallow section, as the diving-wave refracted wavefield typically penetrates to a depth of about one third to one quarter of the recording cable length. For model update below such depths, we need to rely on the reflected wavefield: in the context of FWI, this energy is carried by the cross-talk terms from the RTM imaging condition (e.g. Douma et al., 2010; Jones 2014, Jones 2018). In addition, we employ an extended domain technique to help circumvent the FWI acoustic approximation as well as seeking to explain events not modeled correctly (the extended-source method: Wang et al., 2016; 2017).

From a strategic viewpoint, for the shallow section, we commence deployment of $\mathrm{FWI}$ using the refracted wavefield and a travel-time minimization norm (to avoid cycle skipping), followed by a more conventional leastsquares norm (to optimize resolution). For the deeper structural updates, we then switch to the reflected wavefield using a single-scattering technique (Born modelling) and again employing both the travel-time and then the least-squares norms. Figure 3 outlines the overall generic workflow.

However, it is important to note that due to the various approximations being used within FWI, a purely acoustic data-matching norm does not guarantee that CRP gathers will be flat following migration, as the FWI method only seeks to obtain modelled shot gather that resemble the field data, and does not explicitly seek to ensure postmigration gather flatness. Hence image domain tomography still has a place in the model building workflow in order to help mitigate some of the limiting factors behind FWI, as does the picking of constraint layers at the boundaries of very high velocity contrast interfaces.

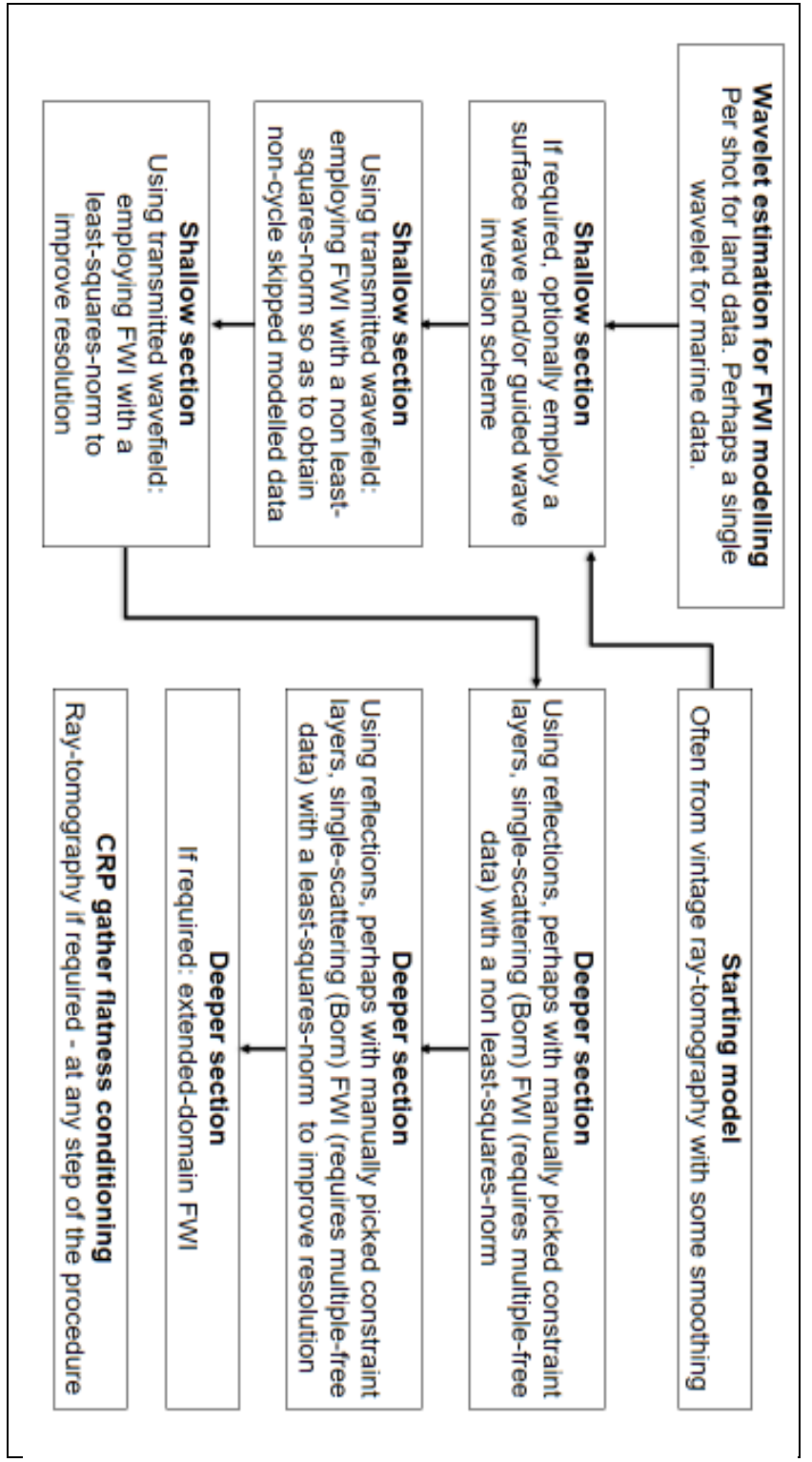

Figure 3. Generic workflow for integration of the various 'flavors' of FWI.

\section{Results}

Figure 4 (top row) shows a comparison for an inline section from a deep water area of the starting model (obtained from structurally constrained ray-tomography) with the final model (refined using FWI for the salt, carbonate, and sub-salt section), along with the associated images, in Figure 4 (centre row) with the velocity overlay, and Figure 4 (bottom row) with the images alone. Figure 5 shows a corresponding crossline secion with model overlay. The most visually pronounced improvements include the flattening of the base-salt and associated simplification of the deeper structural elements. These changes arise thanks to FWI having introduced significant meaningful geological variability in both the salt and carbonate internal velocity distribution, during the FWI model update. 

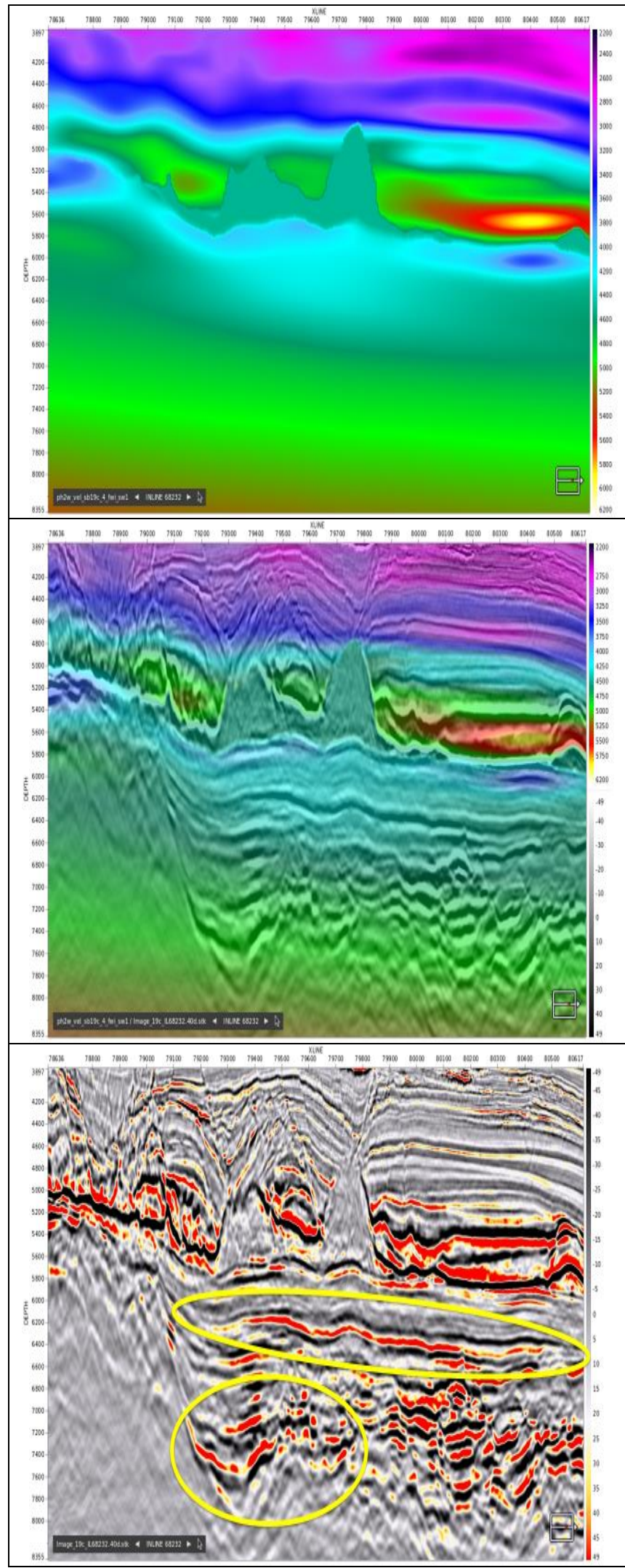

Figure 4: Top row: Left- inline from TTI starting velocity model (from structurally constrained tomography). RightTTI final velocity model.
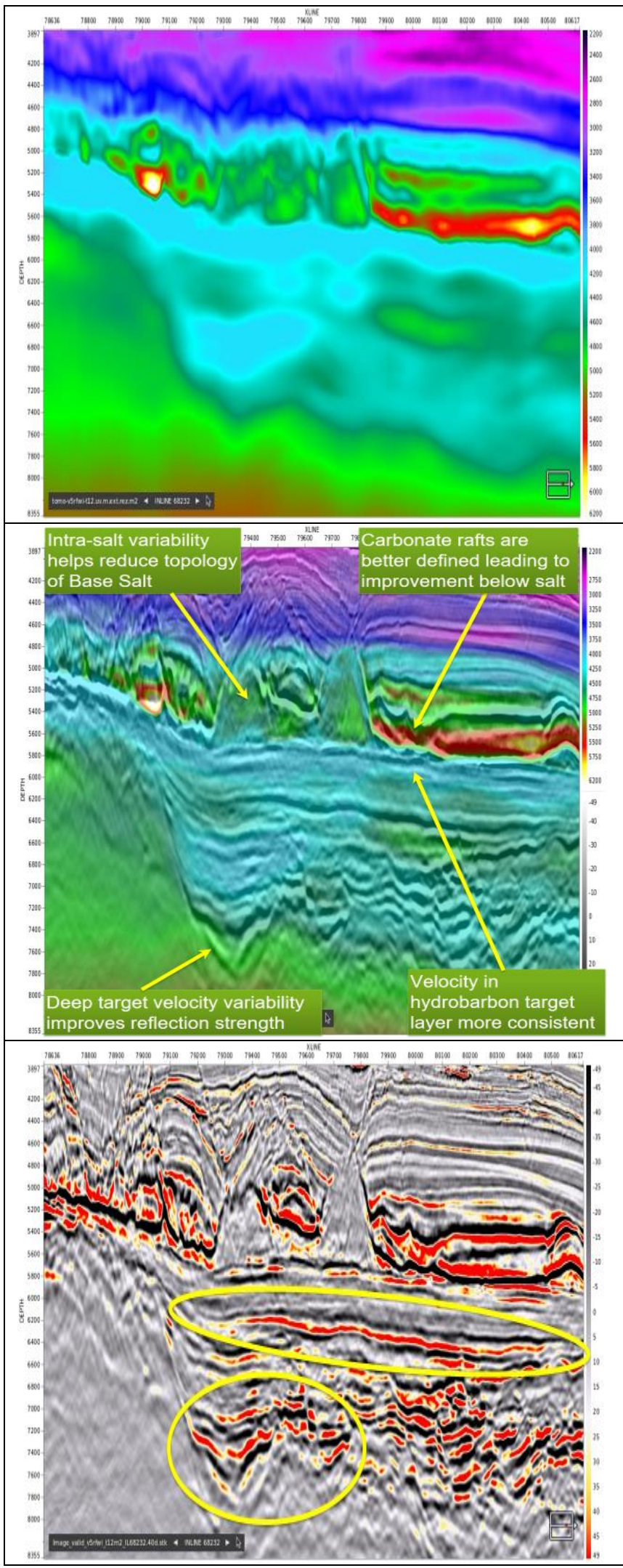

Centre row: Left- TTI starting velocity model and preSDM image. Right- TTI final velocity model and image. Bottom row: Left- starting TTI preSDM image. Right- final TTI image. 


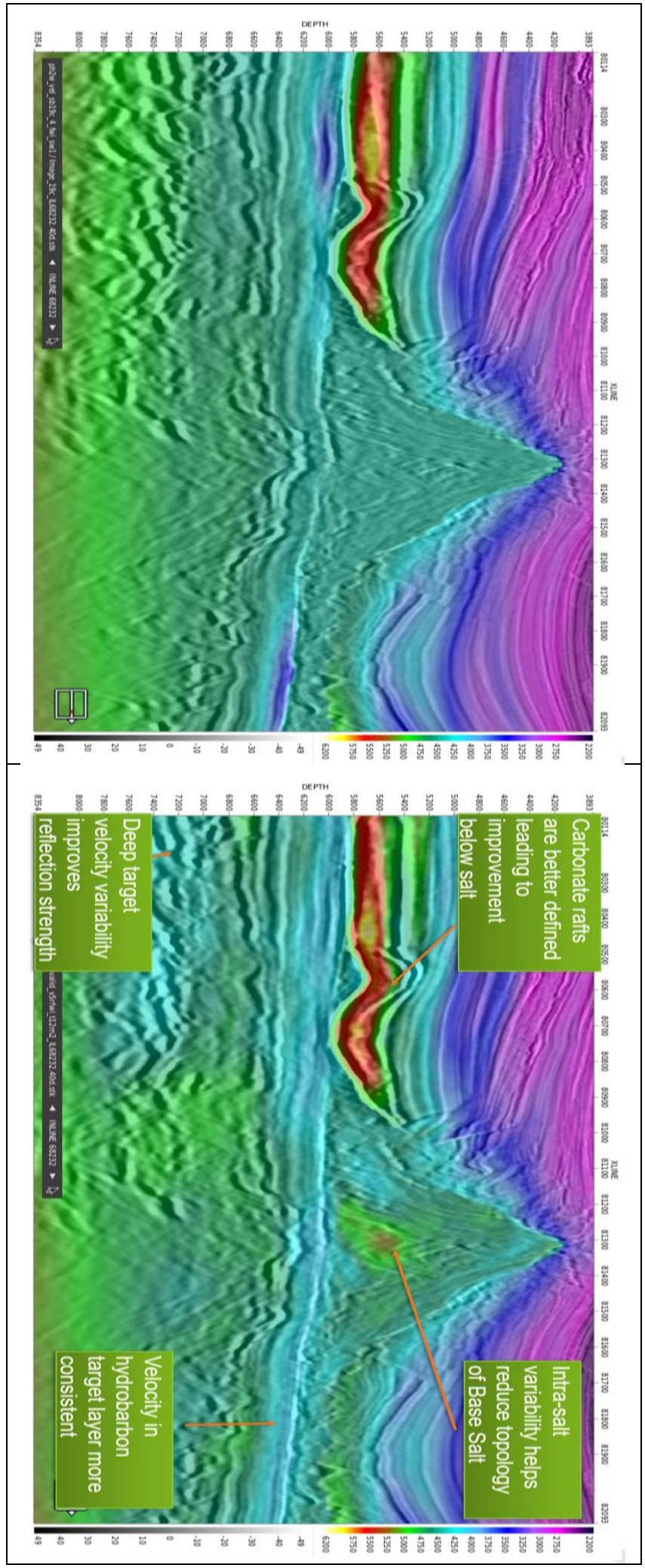

Figure 5 Left- TTI starting velocity model and preSDM image. Right- TTI final velocity model and image. Bottom row: Left- starting TTI preSDM image. Right- final TTI image.

\section{Conclusions}

Large-scale regional model building can be facilitated using a combination of various FWI minimization norms, exploiting both the refracted and reflected wavefields. However, care must be taken so as to avoid using starting models that result in cycle skipping, and final output gathers may need to be produced using models further conditioned using image domain tomography so as to ensure flat image gathers.

\section{Acknowledgements}

We would like to thank Jacques Leveille, for help in preparing this abstract, and to ION for permission to present the work.

\section{References}

Douma, H., D. Yingst, I. Vasconcelos, and J. Tromp, [2010]. On the connection between artifact filtering in reverse-time migration and adjoint tomography: Geophysics, 75, S219-S223.

Jiao, K., D. Sun, X. Cheng, and D. Vigh, [2015]. Adjustive full waveform inversion: 85th Annual International Meeting, SEG, Expanded Abstracts, 1091-1095,

Jones, I.F., [2018]. Velocities, Imaging, and Waveform Inversion (The evolution of characterizing the Earth's subsurface), EET 13, EAGE, 234 pages.

Jones, I.F, [2014], Tutorial: migration imaging conditions. First Break, 32, no.12, 45-55

Jones, I.F, and Davison, I., [2014], Seismic imaging in and around salt bodies. SEG Interpretation, 2, no.4, SL1-SL20.

Pavlov, A, Fruehn J.K., Faw, J., Faw, H., [2018]. Imaging Pitfalls around Salt Domes from the Picanha area, offshore Brazil, EAGE abstracts

Schuster, G.T., [2017]. Seismic Inversion. Investigations in Geophysics \#20, SEG, Tulsa.

Vigh, D., Jiao, K., Cheng, X., Sun, D., Zhang, L.X., [2017]. Seeing below the diving wave penetration with full waveform inversion, $87^{\text {th }}$ Annual International Meeting, SEG, Expanded Abstracts, 1466-1470.

Wang, C., Farmer, P., Yingst, D., Jones, I., Calderon, C., and Brittan, J., [2018]. Traveltime based reflection full waveform inversion, 80 ${ }^{\text {th }}$ Conference \& Exhibition, EAGE, Extended Abstracts, Workshop 1.

Wang, C., Yingst, D., Farmer, P. and Leveille, J. [2016]. Full-waveform inversion with the reconstructed wavefield method. Expanded Abstracts, for the 86th SEG Meeting , Dallas, TX, 1237-1241.

Wang, C., Yingst, D., Farmer, P., Jones, I., Martin, G. and Leveille, J., [2017]. Reconstructed full waveform inversion with the extended source. Expanded Abstracts for the 87th SEG Meeting, FWI5.1.

Warner, M., T. Nangoo, N. Shah, A. Umpleby, J. Morgan, 2013. Full-waveform inversion of cycle-skipped seismic data by frequency down-shifting. $83^{\text {rd }}$ Annual International Meeting, SEG, Expanded Abstracts, 903907

Warner, M. R., and L. Guasch, [2016]. Adaptive waveform inversion: Theory: Geophysics, 81, (6), R429- R445.

Yang, Y., Engquist, B., [2018]. Analysis of optimal transport and related misfit functions in full-waveform inversion, Geophysics, 83, (1), A7-A12. 